\section{(O) OPEN ACCESS}

\title{
Integrated flat detector CT and live fluoroscopic- guided external ventricular drain placement within the neuroangiography suite
}

\author{
David Fiorella, ${ }^{1}$ Lissa Peeling, ${ }^{2}$ Christine M Denice, ${ }^{1}$ Marily Sarmiento, ${ }^{3}$ Henry H Woo ${ }^{1}$
}

\begin{abstract}
- Additional material is published online only. To view please visit the journal online (http://dx.doi.org/10.1136/ neurintsurg-2013-010856).

${ }^{1}$ Department of Neurological Surgery, Stony Brook University Medical Center, Stony Brook, New York, USA

${ }^{2}$ Royal University Hospital, University of Saskatchewan, Saskatoon, Saskatchewan, Canada

${ }^{3}$ Seimens Medical Imaging, Erlangen, Germany
\end{abstract}

\section{Correspondence to} Dr D Fiorella, Department of Neurological Surgery, Stony Brook University Medical Center, Cerebrovascular Center, Health Sciences Center T-12 080, Stony Brook, NY 117948122, USA; david.fiorella@ sbumed.org

Received 7 June 2013 Revised 5 July 2013 Accepted 9 July 2013 Published Online First 26 July 2013

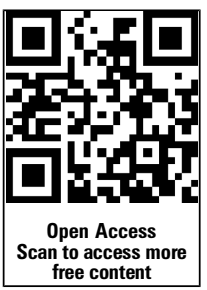

\begin{tabular}{|l|}
\hline To cite: Fiorella D, \\
Peeling L, Denice CM, et al. \\
J Neurolntervent Surg \\
2014;6:457-460. \\
\hline
\end{tabular}

\begin{abstract}
Purpose To demonstrate the feasibility of the application of integrated flat detector (FD) CT and fluoroscopic guidance (iGuide) for the placement of external ventricular drains (EVD) within the neuroangiography suite.

Methods A retrospective review of a prospectively maintained endovascular database identified six patients who underwent EVD placement using iGuide. Patient characteristics, operator, number of passes, accuracy of placement, immediate and delayed periprocedural complications and radiation exposure were assessed. Results Five patients with subarachnoid hemorrhage and one patient with a large cerebellar infarct (average age 45.5 years (range 39-53), four women) underwent EVD placement within the angiography suite using iGuide. Four procedures were performed by a neuroradiologist and two by a neurosurgeon. All catheters were placed with a single pass and all terminated within the frontal horn of the ipsilateral lateral ventricle. No parenchymal or intraventricular hemorrhages were encountered after catheter placement. No patients experienced any immediate or delayed periprocedural complications. Radiation exposure related to the FD CTs required for placement was $593.7 \mathrm{mGy}$ (range 539-673).
\end{abstract}

Conclusions EVD placement under combined CT and fluoroscopic control within the neuroangiography suite is feasible. The technique predictably allows optimized EVD catheter placement with a single pass. We propose that this technique could improve the accuracy, and potentially reduce the complications, of EVD insertion in cerebrovascular patients.

\section{INTRODUCTION}

External ventricular drains (EVDs) have become a standard intervention for the treatment of acutely elevated intracranial pressure resulting from hydrocephalus. ${ }^{1}$ In patients with cerebrovascular pathology, this scenario frequently results from subarachnoid or intraventricular hemorrhage and occasionally from posterior circulation stroke with associated mass effect. Traditionally, EVDs have been placed freehand at the bedside using only external anatomical landmarks for guidance. ${ }^{2}$ Studies assessing freehand EVD placement have reported a wide range of estimates with respect to the accuracy of placement, as well as the rates of associated complications. ${ }^{1-9}$ Many have concluded that imaging guidance has the potential to reduce the number of passes made, improve the accuracy of catheter placement and reduce the associated complications. $^{29}$

We describe our experience using integrated flat detector (FD) CT and live fluoroscopic guidance for EVD placement within the angiography suite in a series of six consecutive patients.

\section{METHODS}

\section{Study patients}

Six patients with cerebrovascular ischemic disease underwent EVD placement using an integrated FD CT and live fluoroscopic guidance system (iGuide; Siemens, Erlangen, Germany). Patient characteristics, operator, number of passes, accuracy of catheter placement, immediate and delayed periprocedural complications and radiation exposure were assessed.

\section{Catheter placement}

The prefrontal area was shaved, prepared and draped in the usual sterile fashion. The insertion point was marked $10 \mathrm{~cm}$ posterior to the glabella and $3 \mathrm{~cm}$ lateral to the midline at the level of the pupillary line. The scalp and periosteum were infiltrated with $1 \%$ lidocaine and a $1 \mathrm{~cm}$ scalp incision was made. Using a standard hemostat, the periosteum subjacent to the incision was then stripped. A self-retaining retractor was either not used or was removed prior to the FD CT to avoid the beam-hardening artifact. A manual twist drill hole (Hand Drill with $5.31 \mathrm{~mm}$ bit; Integra, West Vally City, Utah, USA) was then made. Following placement of the twist drill hole, a cone beam FD CT scan was performed (figure 1A-E). Source data were then reconstructed (Syngo DynaCT; Siemens AG, Forchheim, Germany) into multiplanar images. Reference points were selected on the FD CT images to demarcate the burr hole (catheter entry site) and the targeted point for EVD placement within the frontal horn of the ipsilateral lateral ventricle. Next, the two points were entered into the guidance software (Syngo iGuide; Siemens AG) and a virtual catheter trajectory was superimposed upon the live fluoroscopic image (figure $1 \mathrm{~F}$ ). The fluoroscopic tube was then manipulated into a position that optimally depicted the virtual trajectory and also allowed comfortable EVD insertion by the operator. The dura was punctured using a \#11 scalpel. A $1.9 \mathrm{~mm}$ Bactiseal ventriculostomy catheter (Codman, Raynham, Massachusetts, USA) with stylet was inserted perpendicular to the brain 

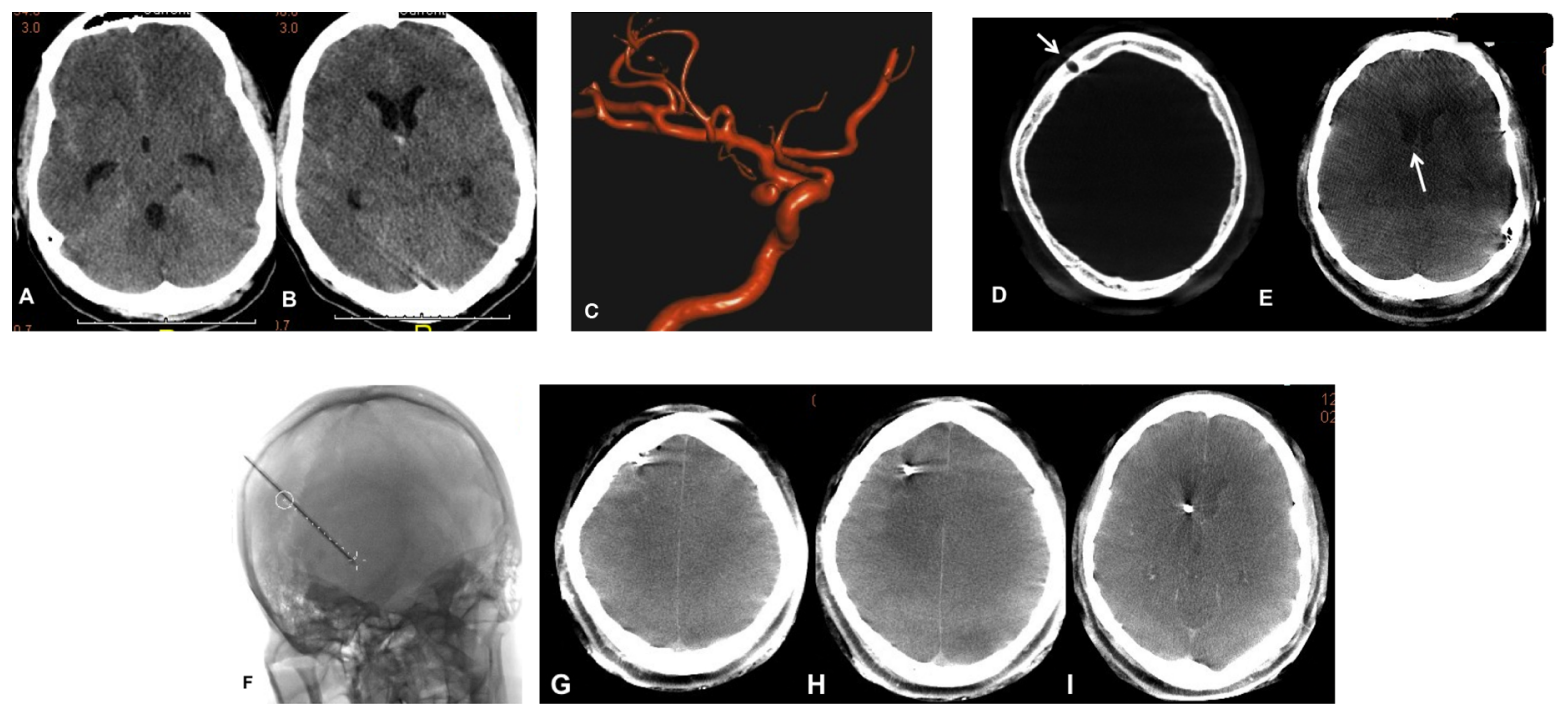

Figure 1 Young patient with acute subarachnoid hemorrhage and mild hydrocephalus. Axial CT images demonstrated diffuse subarachnoid hemorrhage and mild ventriculomegaly ( $A$ and $B$ ). Reconstructed three-dimensional images from rotational angiography source data show a right posterior communicating artery region aneurysm (C). Following burr hole placement, a flat detector (FD), cone beam, CT data set was created and post-processed. The burr hole ( $D$, arrow) and foramen of Monro ( $E$, arrow) are localized on the reconstructed axial images. After registration of the burr hole and the target, the guidance software (iGuide, Siemens Medical Imaging) superimposed an optimal trajectory upon the live fluoroscopic image, which was then used to guide external ventricular drain insertion. The detector was then manipulated into a position which both optimally displayed the catheter trajectory and allowed the operator to comfortably place the catheter. The catheter was then inserted along the mapped trajectory with minor adjustments made to maintain fidelity to the displayed needle path (see online supplementary video). The catheter was advanced to the target, which is demarcated at the terminus of the displayed path (F). The stylet was then removed to allow drainage of cerebrospinal fluid and the catheter was secured into position. A second FD CT was performed, excluding hemorrhage and confirming optimal catheter placement within the ipsilateral frontal horn (G-I).

surface and advanced under live fluoroscopic guidance with fine adjustments made as necessary to preserve fidelity to the virtual trajectory superimposed upon the fluoroscopic image (figure 1, see online supplementary video). When the target was reached, the stylet was removed to allow cerebrospinal fluid drainage. The catheter was then subcutaneously tunneled and secured to the scalp with a 3-0 nylon suture. The incision was closed with staples and the wound was dressed with standard sterile adhesive dressings. Upon completion of the ventriculostomy, a second cone beam FD CT scan (Syngo DynaCT; Siemens AG) was performed to confirm placement and evaluate any associated complications (figure $1 \mathrm{G}-\mathrm{I}$ ).

\section{RESULTS}

Five patients with subarachnoid hemorrhage and one patient with cerebellar infarcts (average age 45.5 years (range 39-53), four women) underwent EVD placement within the angiography suite using iGuide. Four procedures were performed by a neuroradiologist and two by a neurosurgeon. All catheters were placed with a single pass and all terminated within the frontal horn of the ipsilateral lateral ventricle. No new EVD-related parenchymal or intraventricular hemorrhages were detected on the post-procedural FD CT.

One patient went to surgical clipping after completion of EVD placement and diagnostic angiography. One patient underwent diagnostic angiography only. Four patients underwent endovascular aneurysm treatment immediately after ventriculostomy placement: coil embolization $(n=3$, figure 1$)$ or parent artery reconstruction $(n=1)$ with a Pipeline Embolization Device (PED; ev3/Covidien, Irvine, California, USA). All four patients undergoing endovascular therapy were heparinized (mean 5875 Units, range 2000-800) and one also received $7 \mathrm{mg}$ intra-arterial abciximab following PED placement.
No patients experienced any immediate or delayed periprocedural complications. Total radiation exposure related to the two FD CTs required for the delineation of the optimized trajectory (pre-insertion) and subsequent evaluation (post-insertion) was 593.7 mGy (range 539-673).

\section{DISCUSSION}

The present case series demonstrates the feasibility of using FD imaging guidance for the placement of EVDs in the neuroangiography suite. The integrated FD CT-based live fluoroscopic guidance system (iGuide) reproducibly allowed efficient, accurate and predictable ventriculostomy placement in all patients with a single catheter pass. It is possible that a reduction in the number of passes and an improvement in the accuracy of catheter placement could improve the safety and efficacy of the procedure in appropriately selected patients.

EVD placement has been established as a standard procedure for the temporary diversion of cerebrospinal fluid in the setting of acute hydrocephalus. EVD insertion has traditionally been performed without imaging guidance, as a freehand procedure. A number of studies have evaluated the accuracy and complication rates associated with freehand EVD placement. ${ }^{1-11}$

Estimates of the accuracy of EVD placement have varied widely and are partly dependent on the definitions used. ${ }^{2}$ Rates of misplacement ranging between $7 \%$ and $45 \%$ have been reported. ${ }^{2}{ }^{12}$ Huyette et al reported a 22.4\% rate of extraventricular catheter placement in a series of 97 patients. In this study, an average of two passes were required for each successful placement. ${ }^{13}$ Abdoh et al ${ }^{2}$ evaluated EVD trajectories and reported that less than half were placed with an angular variation of $\pm 5^{\circ}$ to the targeted ipsilateral foramen of Monro. These authors concluded that freehand placement of EVDs lacked sufficient accuracy. Toma et al ${ }^{9}$ reviewed 183 post-EVD 
placement scans and found that only $40 \%$ had been placed within the ipsilateral frontal horn. Of those not accurately placed, 40\% ultimately required revision. These authors also concluded that freehand EVD placement is an inaccurate procedure and urged studies to assess the use of neuronavigation to guide placement. In surveys of practicing neurosurgeons and residents, respondents admitted to often requiring multiple catheter passes to achieve successful EVD placement as well as frequent catheter misplacement. ${ }^{14} 15$

While authors have concluded that freehand EVD insertion is a safe procedure, many have acknowledged that the rates of complications are higher than anticipated. Maniker et $a l^{8}$ reported a 33\% hemorrhage rate following EVD placement and noted that the incidence in patients with cerebrovascular disease was higher than in those without (39\% vs 23\%). Similarly, Gardner et $a l^{4}$ reported a $41 \%$ rate of hemorrhage after EVD insertion in a series of 188 patients. While the majority of the hemorrhages observed in these series were small and deemed not to be clinically significant, $10 \%$ of patients in the series of Gardner et al had hemorrhages that were $>15 \mathrm{~mL}^{4}$ In addition, although not clinically significant initially, even small hemorrhages may create foci for seizure generation or may represent a relative or absolute contraindication to anticoagulation.

Thus, while freehand EVD placement is an important and often lifesaving procedure, the accuracy and possibly the safety of the procedure could potentially be improved in scenarios in which imaging guidance is feasible. Theoretically this is of greatest relevance to patients with cerebrovascular disease for a number of reasons. First, as demonstrated in our series, patients often require anticoagulation and/or antiplatelet medications immediately after EVD placement during subsequent endovascular treatment of the index hemorrhagic lesion. While previous studies have indicated that endovascular treatment (and heparinization) is relatively safe after freehand EVD placement, it stands to reason that it is preferential to have an accurately placed EVD with a single pass in these scenarios. ${ }^{5}$ Second, acute intracranial hemorrhage may create mass effect and shift, resulting in a distortion of the ventricular system, which is challenging to account for during freehand placement but presents no significant technical impediment in the setting of imaging guidance. Third, most patients with cerebrovascular disease are transported to the neuroangiography suite at an early point in their care for diagnosis and often treatment. As such, using the guidance mechanisms available within the neuroangiography suite is a seamless extension of the application of the imaging equipment for the patient's treatment. The overall excess radiation exposure required for the procedure is very low compared with freehand EVD placement-essentially equivalent to one additional basic head CT study. The time expenditure required is also minimal. The FD CT imaging and post-processing required to delineate the optimal catheter path for live fluoroscopic guidance can be obtained in $<5 \mathrm{~min}$. In many cases this time expenditure can be offset by facilitating successful EVD placement with a single pass. Fourth, in the setting of large-volume intraventricular hemorrhage, targeted catheter placement may optimize the efficacy of intraventricular hemorrhage clearance, particularly if intraventricular thrombolysis is performed. ${ }^{10} 16$ Finally, Maniker et $a l^{8}$ demonstrated that the most reliable predictive factor for hemorrhage (and larger sized hemorrhage) after EVD placement was cerebrovascular disease. For this reason, a guidance technique with the potential to minimize the need for multiple passes could reduce hemorrhage rates in this higher risk cohort.
There are important limitations of the current case series and the technique described. First, this is a small retrospective series compiled to demonstrate feasibility and, as such, no conclusions can be made regarding efficacy and improved safety in comparison with freehand EVD placement. This initial feasibility pilot will provide a basis for a single-arm prospective trial to confirm safety and provide a more accurate assessment of efficacy. Second, some patients may not be appropriate candidates for this technique. Those who are acutely decompensated might not have sufficient time to be transported to the neuroangiography suite for image-guided EVD placement. Also, patients who are unable to remain still between the initial registration DynaCT and subsequent EVD placement are not suitable candidates. Any change in the head position between the initial scan and subsequent live fluoroscopic guidance could result in significant misregistration and subsequent catheter misplacement. For this reason, patients who are intubated and sedated represent the best candidates for the guidance technique described. Third, no intravenous or intra-arterial contrast was injected at the time of the initial DynaCT. It is possible that the iGuide technique could be further optimized by performing the DynaCT examination after contrast injection to delineate any large regional vessels in the vicinity of the twist drill hole. Finally, while the accuracy of DynaCT has been validated for the detection of parenchymal hemorrhage, the level of contrast resolution using the present version of the software is still below that of conventional CT. ${ }^{17}$ It is therefore possible that some small pericatheter hemorrhages could have been missed on the immediate postcatheter placement DynaCT.

\section{CONCLUSION}

FD CT-based live fluoroscopic guidance represents a feasible means by which to reproducibly and accurately place ventriculostomy catheters with a single pass within the neuroangiography suite.

Contributors All authors have made substantial contributions to the conception and design, acquisition of data, or analysis and interpretation of data.

Funding State University of NY at Stony Brook receives research grants from Siemens Medical Imaging.

\section{Competing interests}

Ethics approval Ethical approval for this retrospective case series was obtained from the Institutional Review Board.

Provenance and peer review Not commissioned; externally peer reviewed.

Open Access This is an Open Access article distributed in accordance with the Creative Commons Attribution Non Commercial (CC BY-NC 3.0) license, which permits others to distribute, remix, adapt, build upon this work non-commercially, and license their derivative works on different terms, provided the original work is properly cited and the use is non-commercial. See: http://creativecommons.org/licenses/by-nc/3.0/

\section{REFERENCES}

1 Gigante P, Hwang BY, Appelboom G, et al. External ventricular drainage following aneurysmal subarachnoid haemorrhage. Br J Neurosurg 2010;24:625-32.

2 Abdoh MG, Bekaert 0, Hodel J, et al. Accuracy of external ventricular drainage catheter placement. Acta Neurochir 2012;154:153-9.

3 Ehtisham A, Taylor S, Bayless $L$, et al. Placement of external ventricular drains and intracranial pressure monitors by neurointensivists. Neurocrit Care 2009;10:241-7.

4 Gardner PA, Engh J, Atteberry D, et al. Hemorrhage rates after external ventricular drain placement. J Neurosurg 2009;110:1021-5.

5 Hoh BL, Nogueira RG, Ledezma CJ, et al. Safety of heparinization for cerebral aneurysm coiling soon after external ventriculostomy drain placement. Neurosurgery 2005:57:845-9.

6 Hsieh CT, Chen GJ, Ma HI, et al. The misplacement of external ventricular drain by freehand method in emergent neurosurgery. Acta Neurol Belg 2011;111:22-8.

7 Kosty J, Pukenas B, Smith M, et al. latrogenic vascular complications associated with external ventricular drain placement: a report of 8 cases and review of the literature. Neurosurgery 2013;72(2 Suppl Operative):ons208-13. 
8 Maniker AH, Vaynman AY, Karimi RJ, et al. Hemorrhagic complications of external ventricular drainage. Neurosurgery 2006;59(4 Suppl 2):ONS419-24.

9 Toma AK, Camp S, Watkins LD, et al. External ventricular drain insertion accuracy: Is there a need for change in practice?. Neurosurgery 2009;65:1197-200.

10 Jaffe J, Melnychuk E, Muschelli J, et al. Ventricular catheter location and the clearance of intraventricular hemorrhage. Neurosurgery 2012;70:1258-63.

11 Struffert T, Eyupoglu IY, Huttner HB, et al. Clinical evaluation of flat-panel detector compared with multislice computed tomography in 65 patients with acute intracranial hemorrhage: initial results. Clinical article. J Neurosurg 2010;113:901-7.

12 Kakarla UK, Kim LJ, Chang SW, et al. Safety and accuracy of bedside external ventricular drain placement. Neurosurgery 2008;63(1 Suppl 1):ONS162-6; discussion ONS166-7
13 Huyette DR, Turnbow BJ, Kaufman C, et al. Accuracy of the freehand pass technique for ventriculostomy catheter placement: retrospective assessment using computed tomography scans. J Neurosurg 2008;108:88-91.

14 O'Neill BR, Velez DA, Braxton EE, et al. A survey of ventriculostomy and intracranial pressure monitor placement practices. Surg Neurol 2008:70:268-73.

15 Rehman T, Rehman AU, Rehman A, et al. A US-based survey on ventriculostomy practices. Clin Neurol Neurosurg 2012;114:651-4.

16 Dey $\mathrm{M}$, Jaffe J, Stadnik $A$, et al. External ventricular drainage for intraventricular hemorrhage. Curr Neurol Neurosci Rep 2012;12:24-33.

17 Struffert T, Richter G, Engelhorn T, et al. Visualisation of intracerebral haemorrhage with flat-detector CT compared to multislice CT: results in 44 cases. Eur Radiol 2009;19:619-25 